Table S4: mutaions between M5 and 16M in virulence associated genes

\begin{tabular}{|c|c|c|c|c|c|c|}
\hline Functional group & Gene & ORF of $16 \mathrm{M}$ & ORF of M5 & SNP & Insersion & Deletion \\
\hline \multicolumn{7}{|l|}{$\begin{array}{l}\text { "Classical" virulence factors } \\
\text { Evelope molecules }\end{array}$} \\
\hline \multirow[t]{12}{*}{ Lipopolysaccharide } & IpsB & BMEI0509 & M5GL002903 & A1044G & & \\
\hline & IpsA & BMEI1326 & M5GL001479 & C278T(S93L) & & \\
\hline & $w b d A$ & BMEI0997 & M5GL000999 & $\begin{array}{l}\text { T347C(L116S) } \\
\text { G1103C(R368P) }\end{array}$ & & \\
\hline & $w b p Z$ & BMEI1393 & M5GL001558 & G942A & & \\
\hline & $p m m$ & BMEI1396 & M5GL001561 & $\begin{array}{l}\text { T397C(F133L) } \\
\text { A903G }\end{array}$ & & \\
\hline & $w b k A$ & BMEI1404 & M5GL001566 & & & \\
\hline & $r f b D$ & BMEI1413 & M5GL001572 & & & \\
\hline & perA & BMEI1414 & M5GL001573 & & & \\
\hline & $w b p L$ & BMEI1426 & M5GL001578 & T1004G(L335R) & & \\
\hline & pgm & BMEI1886 & M5GL002265 & A752G( H251R) & & \\
\hline & $\operatorname{man} B$ & BMEII0899 & M5GL000375 & $\mathrm{C} 279 \mathrm{~T}$ & & \\
\hline & wbpW & BMEII0900 & M5GL000393 & & & \\
\hline \multirow[t]{7}{*}{ Outer membrance protein } & omp19 & BMEI0135 & M5GL001873 & & & \\
\hline & omp25 & BMEI1249 & M5GL001304 & T276C & & \\
\hline & omp10 & BMEII0017 & M5GL002026 & & & \\
\hline & $m g t A$ & BMEI0271 & M5GL003111 & $\begin{array}{l}\text { C142T(P48S) } \\
\text { T252C } \\
\text { G380A(R127K) }\end{array}$ & & \\
\hline & upps & BMEI0827 & M5GL002568 & & & \\
\hline & amic & BMEI1056 & M5GL001096 & $\begin{array}{l}\text { A96C } \\
\text { A545T(N182I) }\end{array}$ & & \\
\hline & dacF & BMEII0350 & M5GL001341 & $\mathrm{G} 1261 \mathrm{~A}(\mathrm{E} 421 \mathrm{~K})$ & & \\
\hline \multicolumn{7}{|l|}{ Secretion or transport system } \\
\hline T4SS & $\operatorname{virB1}$ & BMEII0025 & M5GL000739 & & & \\
\hline & virB2 & BMEII0026 & M5GL000746 & $\begin{array}{l}\text { G54A } \\
\text { G270C }\end{array}$ & & \\
\hline & virB3 & BMEII0027 & M5GL000747 & & & \\
\hline & virB4 & BMEII0028 & M5GL000748 & A463C(K155Q) & & \\
\hline
\end{tabular}




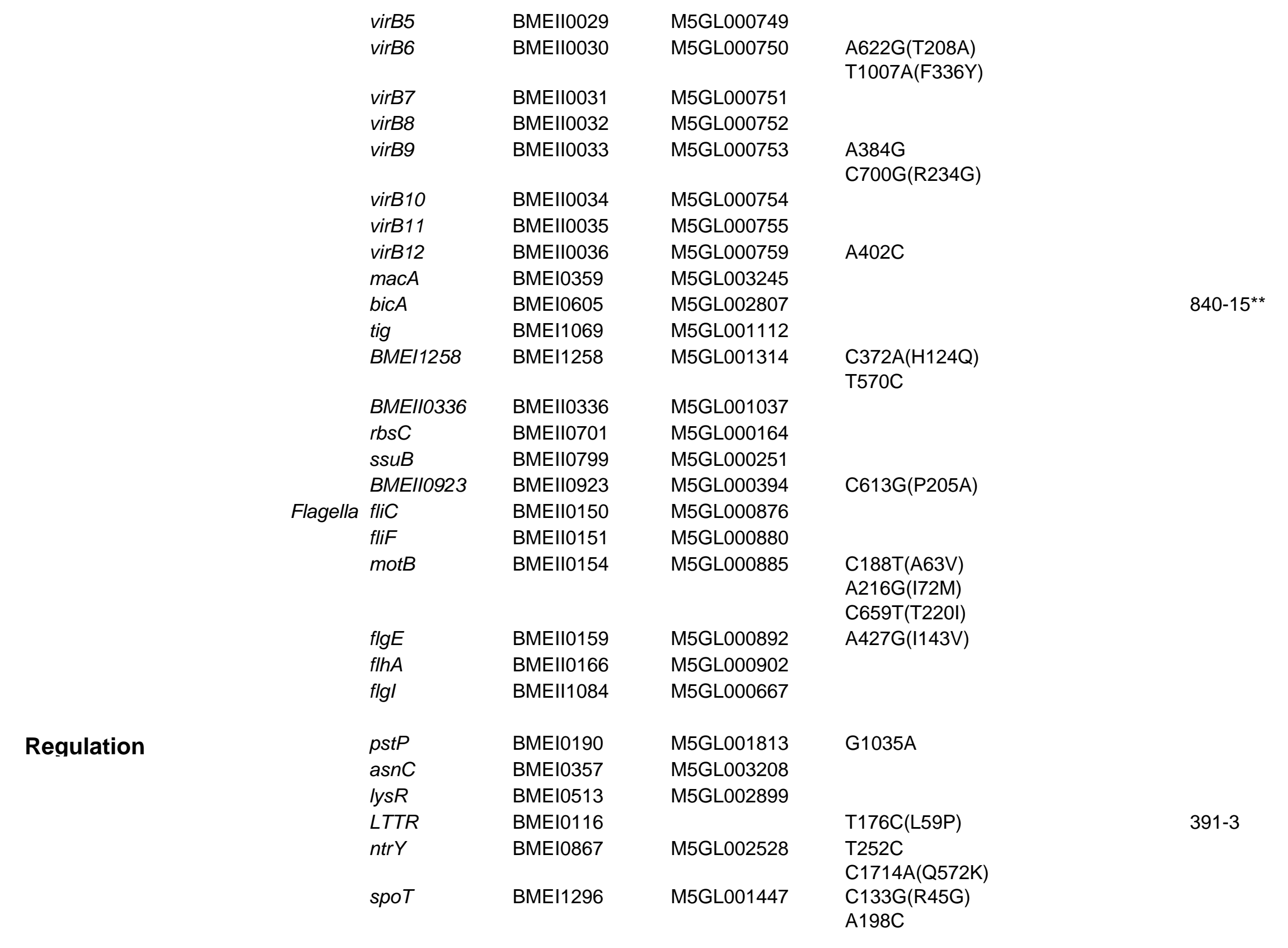




\begin{tabular}{|c|c|c|c|c|}
\hline & & & & \\
\hline & feuQ & BMEI1336 & M5GL001489 & \\
\hline & feuP & BMEI1337 & M5GL001490 & G328A(A110T) \\
\hline & $v s r B$ & BMEI1606 & M5GL001756* & \\
\hline & $g \ln L$ & BMEI1786 & M5GL002371 & \\
\hline & bvrS & BMEI2035 & end of contig56 & \\
\hline & $b v r R$ & BMEI2036 & M5GL002033 & G300T \\
\hline & nodV & BMEII0052 & M5GL000775 & $\begin{array}{l}\text { G465A } \\
\text { T530C(V177A) }\end{array}$ \\
\hline & RpiR & BMEII0573 & M5GL000054 & \\
\hline & $\operatorname{divK}$ & BMEII0659 & M5GL000112 & \\
\hline & gntR & BMEII1066 & M5GL000649 & \\
\hline & deoR & BMEII1093 & M5GL000676 & \\
\hline & $v j b R$ & BMEII1116 & M5GL000698 & \\
\hline Metal acquisition & mgtB & BMEII0056 & M5GL000797 & C921T \\
\hline & $d h b C$ & BMEII0077 & M5GL000809 & $\begin{array}{l}\text { A196G(K66E) } \\
\text { G576A(M192I) }\end{array}$ \\
\hline & znuC & BMEII0177 & M5GL000903 & A149C(Q50P) \\
\hline & znuA & BMEII0178 & M5GL000502 & C250T(L84F) \\
\hline & $n i k A$ & BMEII0487 & M5GL002228 & $\begin{array}{l}\text { T242C(F81S) } \\
\text { A531G }\end{array}$ \\
\hline & fbpA & BMEII0584 & M5GL000066 & \\
\hline Amino acid metabolism & & & & \\
\hline Sythesis & IysA & BMEI0084 & M5GL001924 & $\begin{array}{l}\text { C739G(P247D) } \\
\text { C740A(P247D) } \\
\text { G1181T(G394V) }\end{array}$ \\
\hline & leuC & BMEI0157 & M5GL001848 & \\
\hline & leuA & BMEI0451 & M5GL002967 & G878T (G293V) \\
\hline & $\operatorname{car} A B$ & BMEI0526 & M5GL002888 & $\mathrm{G} 484 \mathrm{~A}(\mathrm{~A} 162 \mathrm{~T})$ \\
\hline & $\operatorname{ser} B$ & BMEI0615 & M5GL002796 & G712C(V238L) \\
\hline & $i / v I$ & BMEI0617 & M5GL002793 & T684C \\
\hline & $i l v C$ & BMEI0624 & M5GL002785 & $\mathrm{T} 162 \mathrm{C}$ \\
\hline & thrA & BMEI0725 & M5GL002681 & C1269G(D423E) \\
\hline & cysK & BMEI0933 & M5GL000934 & T308C(V103A) \\
\hline
\end{tabular}




\begin{tabular}{|c|c|c|c|c|c|}
\hline & thrC & BMEI1450 & M5GL001603 & G1323A & \\
\hline & aroc & BMEI1506 & M5GL001656 & C684T & \\
\hline & & & & G946A(D316N) & \\
\hline & hisD & BMEI1668 & M5GL002448 & & \\
\hline & metH & BMEI1759 & M5GL002400 & G523C(V175L) & \\
\hline & & & & C2041T(R681W) & \\
\hline & & & & C2924T(P975L) & \\
\hline & & & & C3375T & \\
\hline & $i / v D$ & BMEI1848 & M5GL002306 & $\mathrm{A} 1235 \mathrm{G}(\mathrm{H} 412 \mathrm{R})$ & \\
\hline & hisF & BMEI2041 & M5GL002031 & & \\
\hline & glt1 & BMEII0039 & M5GL000760 & & \\
\hline & gltD & BMEII0040 & M5GL000771 & G1659A & \\
\hline & & & & C1767T & \\
\hline & & & & C2058A & \\
\hline & & & & С3855T & \\
\hline Degradation & gtyA & BMEI1192 & M5GL001243 & & $560-1$ \\
\hline & & & & & $753-562$ \\
\hline & $x f p$ & BMEII0881 & M5GL000314 & & \\
\hline Transport & livH & BMEI0258 & M5GL003098 & G449T(G150V) & \\
\hline & & & & C909A(D303E) & \\
\hline & $d p p A$ & BMEI0433 & M5GL002985 & A35G & \\
\hline & artl & BMEI1104 & M5GL001151 & & \\
\hline & bacA & BMEl1553 & end of contig46 & & \\
\hline Unknown & aspC & BMEI0516 & M5GL002897 & A450G & \\
\hline & $a s p B$ & BMEI0626 & M5GL002784 & & \\
\hline Catabolism & pyc & BMEI0266 & M5GL003106 & T135C & \\
\hline & & & & C156T & \\
\hline & & & & C186T & \\
\hline & & & & C885T & \\
\hline & & & & T1358A(L453Q) & \\
\hline & & & & A2846G(D949G) & \\
\hline & & & & G3220A(G1074S) & \\
\hline & gloA & BMEI0730 & M5GL002674 & & \\
\hline & $c b b E$ & BMEI1116 & M5GL001163 & & \\
\hline & gale & BMEI1237 & M5GL001290 & & \\
\hline
\end{tabular}




\begin{tabular}{|c|c|c|c|c|c|c|}
\hline & $p g i$ & BMEI1636 & M5GL001786 & & & \\
\hline & rbsk & BMEII0089 & M5GL000852 & G369C & & \\
\hline & eryc & BMEII0428 & M5GL001391 & G270A & & \\
\hline & ery $B$ & BMEII0429 & M5GL002174 & & & \\
\hline & galcD & BMEII0485 & M5GL002175 & $\mathrm{C} 672 \mathrm{~T}$ & & \\
\hline & $z w f$ & BMEII0513 & M5GL002230 & & & \\
\hline & $\operatorname{mocC}$ & BMEII0570 & M5GL003242 & & & \\
\hline & glpK & BMEII0823 & M5GL000289 & & & \\
\hline & gnd & BMEII1124 & M5GL000706 & T267C & & \\
\hline & & & & T834G & & \\
\hline & & & & A905C(D302A) & & \\
\hline & $\operatorname{mos} A$ & BMEI1301 & M5GL001452 & T120A & & \\
\hline & & & & A445G(I149V) & & \\
\hline & $n d v B$ & BMEI1837 & M5GL002321 & $\mathrm{C} 2556 \mathrm{~T}$ & $3638-1$ & $3631-1$ \\
\hline & & & & A2784G & & \\
\hline & & & & A3587C(D1196A) & & \\
\hline & & & & T3588A(D1196A) & & \\
\hline & & & & C3589T(H1197S) & & \\
\hline & & & & A3590C(H1197S) & & \\
\hline & & & & T3594C & & \\
\hline & & & & C3774G(G1258E) & & \\
\hline & & & & G3775C(G1259R) & & \\
\hline & & & & T3780G & & \\
\hline & & & & C3782G(P1261R) & & \\
\hline & & & & C4638T & & \\
\hline & & & & G4996A(A1666T) & & \\
\hline & & & & G6600T(E2200D) & & \\
\hline & & & & C7557G & & \\
\hline & gtrB & BMEII1101 & M5GL000684 & A95G(N32S) & & \\
\hline Transport & $\operatorname{mos} C$ & BMEI0267 & M5GL003107 & & & \\
\hline & malk & BMEI1713 & M5GL002417 & C1026T & & \\
\hline & exsA & BMEI1742 & M5GL002418 & & & \\
\hline & $d b s A$ & BMEII0300 & M5GL001018 & G1668A & & $1-1053$ \\
\hline & & & & G1714A(G572R) & & \\
\hline & $\operatorname{araG}(g g u A)$ & BMEII0361 & M5GL001357 & & & \\
\hline & ugpA & BMEII0591 & M5GL000071 & & & \\
\hline
\end{tabular}




$\begin{array}{llll}\text { ugpA } & \text { BMEII0624 } & \text { M5GL000080 } & \\ \text { gluP } & \text { BMEII1053 } & \text { M5GL000635 } & \text { C50T(S17F) } \\ & & & \text { C104G(S35W) }\end{array}$

\section{DNA/RNA metabolism}

\begin{tabular}{|c|c|c|c|c|}
\hline \multirow[t]{16}{*}{ Synthesis } & hpt & BMEI0082 & M5GL001926 & \\
\hline & purE & BMEI0296 & M5GL003138 & \\
\hline & $d u t$ & BMEI0358 & M5GL003209 & \\
\hline & pth & BMEI0480 & M5GL002936 & G222A \\
\hline & $\operatorname{mia} A$ & BMEI0616 & M5GL002795 & T629A(V210E) \\
\hline & purL & BMEI1127 & M5GL001174 & $\begin{array}{l}\text { T918C } \\
\text { A1483C }(T 495 P) \\
\text { A1489C }(T 497 P)\end{array}$ \\
\hline & purM & BMEI1240 & M5GL001294 & $\begin{array}{l}\text { G189A } \\
\text { T339C }\end{array}$ \\
\hline & purN & BMEI1241 & M5GL001295 & \\
\hline & pyrc & BMEI1281 & M5GL001432 & \\
\hline & purF & BMEI1488 & M5GL001638 & \\
\hline & purD & BMEI1519 & M5GL001668 & $\begin{array}{l}\text { T95C(L32P) } \\
\text { G919A(V307I) }\end{array}$ \\
\hline & pyrD & BMEI1611 & M5GL001761 & A138G \\
\hline & pyrc & BMEII0669 & M5GL000151 & \\
\hline & pyrB & BMEII0670 & M5GL000162 & \\
\hline & $n d r l$ & BMEII0931 & M5GL000414 & \\
\hline & $n r d H$ & BMEII0932 & M5GL000422 & \\
\hline \multirow[t]{7}{*}{ Catabolism } & $x \operatorname{se} A$ & BMEII0527 & M5GL003227 & $\begin{array}{l}\text { C810T } \\
\text { A1416C } \\
\text { G1465A9V489I) }\end{array}$ \\
\hline & xerD & BMEI0040 & M5GL001969 & T90C \\
\hline & alkA & BMEI0382 & M5GL003274 & \\
\hline & $\operatorname{rec} A$ & BMEI0787 & M5GL002614 & \\
\hline & $\operatorname{rps} A$ & BMEI1915 & M5GL002152 & G240A \\
\hline & mutM & BMEI1946 & M5GL002123 & \\
\hline & aidB & BMEII0671 & M5GL000163 & \\
\hline
\end{tabular}


rpoA

BMEI0781

M5GL002622

C183T

tldD

BMEI1468

M5GL001622

G855T

\section{Vitamines}

Icofacteurs

$c o b B$

BMEI0705

dxps

hemH

cobW

pncA

BMEI1498

BMEII0018

BMEII0308

BMEI0545

\section{ppiD}

BMEI0845

IChaperones

\section{Oxidoreduction}

\begin{tabular}{|c|c|c|c|}
\hline$h f q$ & BMEI0872 & M5GL002524 & \\
\hline Ion & BMEI0876 & M5GL002520 & T2109C \\
\hline htrA & BMEI1330 & M5GL001483 & \\
\hline dnaJ & BMEI1513 & M5GL001664 & $\begin{array}{l}\text { A303C } \\
\text { G398A(G133D) } \\
\text { A555G }\end{array}$ \\
\hline dnaK & BMEI2002 & M5GL002066 & A37C(M13L) \\
\hline dsbB domain & BMEI0384 & M5GL003276 & G120A(M40I) \\
\hline $\operatorname{trkH}$ & BMEI0512 & M5GL002900 & \\
\hline caiB domain & BMEI0898 & M5GL002498 & $\begin{array}{l}\text { G201T(W67C) } \\
\text { G552A }\end{array}$ \\
\hline dsbA domain & BMEI1040 & M5GL001080 & T726C \\
\hline
\end{tabular}

C442T(L148F)

G567A

C483T

C1028T(P343L) 735-15

C237T

A1006C(M336L)

M5GL002549

M5GL002524

M5GL002520

M5GL001483

M5GL001664

G398A(G133D)

A555G

G120A(M40I)

G552A 


\begin{tabular}{|c|c|c|c|c|c|}
\hline dsbA domain & BMEI1060 & M5GL001101 & T483C & & \\
\hline dsbA domain & BMEI1440 & M5GL001592 & & & \\
\hline cysl & BMEI1766 & M5GL002393* & & & $\begin{array}{l}1504-4 \\
1672-88\end{array}$ \\
\hline$c y c Y$ & BMEI1849 & M5GL002305 & & & \\
\hline$f d h A$ & BMEII0378 & M5GL001371 & $\begin{array}{l}\text { G1112A(R371Q) } \\
\text { G1164A(M388I) } \\
\text { C1343T(A448V) } \\
\text { G1598A(R533H) } \\
\text { T2139C }\end{array}$ & & \\
\hline sodC & BMEII0581 & M5GL000057 & & & \\
\hline $\operatorname{cydB}$ & BMEII0759 & M5GL000197 & & & \\
\hline cydc & BMEII0761 & M5GL000248 & C1404G & & $809-12$ \\
\hline$c y d D$ & BMEII0762 & M5GL000250 & & & \\
\hline narG & BMEII0950 & M5GL000529* & & $0-851$ & $72-1,176-1$ \\
\hline nore & BMEII1001 & M5GL000584 & & & \\
\hline caiB domain & BMEII1019 & M5GL000603 & T686C(V229A) & & \\
\hline$g \ln A$ & BMEI0979 & M5GL000982 & T1075G(F359V) & & \\
\hline nifS & BMEI1043 & M5GL001083 & C274T(P92S) & & \\
\hline$g \ln D$ & BMEI1804 & M5GL002353 & $\begin{array}{l}\text { T427C(Y143H) } \\
\text { G627C } \\
\text { T2544C }\end{array}$ & & \\
\hline $\begin{array}{l}\text { Tetratricopepti } \\
\text { de repeat } \\
\text { family protein }\end{array}$ & BMEI1531 & M5GL001680 & & & \\
\hline pheB & BMEII0136 & M5GL000874 & T271C(F91L) & & \\
\hline \multirow[t]{5}{*}{ Zinc protease } & BMEII1037 & M5GL000620 & C154T(P52S) & & \\
\hline & BMEI0085 & M5GL001923 & & & \\
\hline & BMEI0455 & M5GL002963 & G500T(R167L) & & \\
\hline & BMEI0671 & M5GL002741 & $\begin{array}{l}\text { C226T(R76C) } \\
\text { T960C } \\
\text { T1065C }\end{array}$ & & \\
\hline & BMEI1229 & M5GL001281 & & & \\
\hline
\end{tabular}




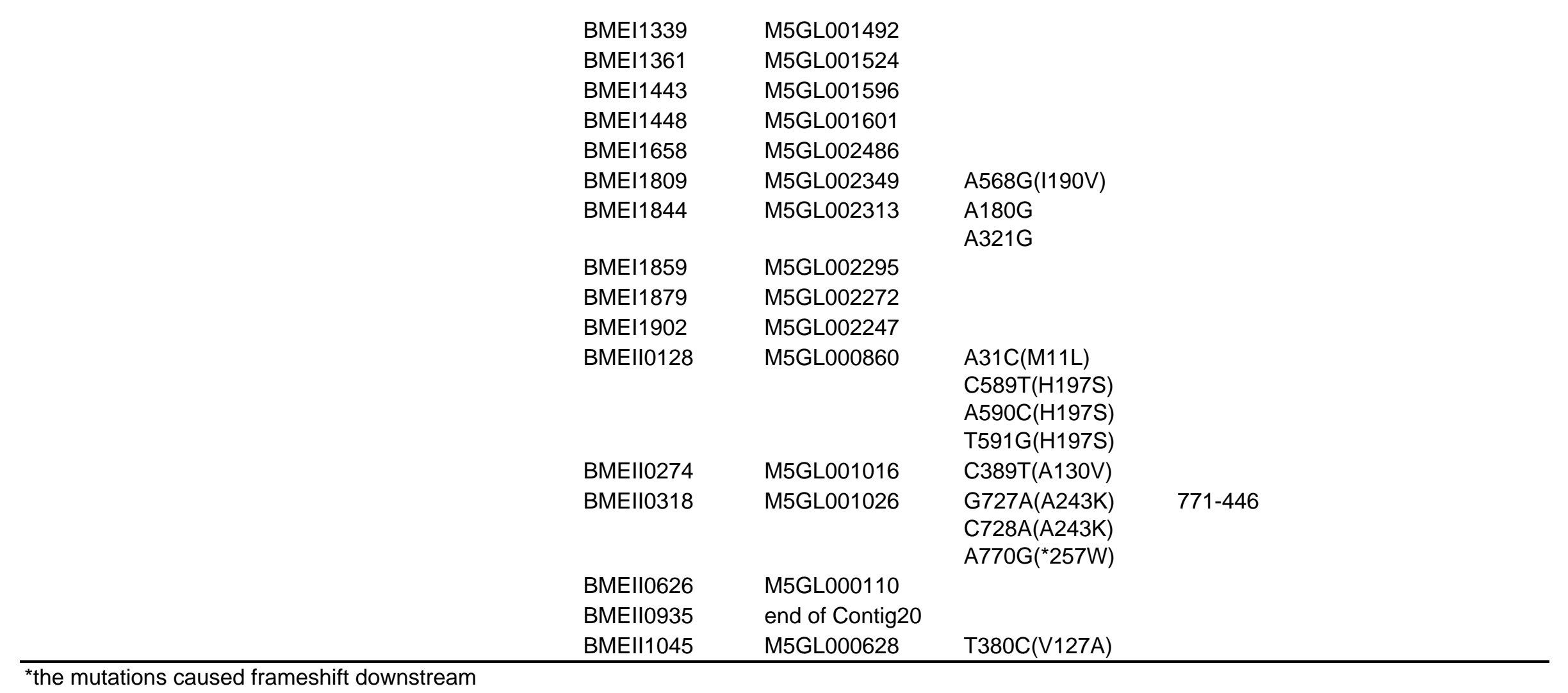

*the mutations caused frameshift downstream

**location of indel - length of fragment 